mother of the 2 siblings presented a history of multiple spontaneous abortions.

The management strategy of the patients herein was flexible due to unpredictable pathologic and clinical evolution of the tumor. At birth, PGE1 therapy and adequate atrial-level shunting, requiring atrial septostomy in 1 patient, were necessary due to univentricular physiology with ductal-dependent pulmonary blood flow. Unexpectedly, both neonates presented severe pulmonary hypertension, only partially responsive to intravenous and inhaled pulmonary vasodilators. Prematurity and persistent fetal circulation were thought to be responsible; only after postmortem diagnosis of patient 2 did we identify recurrent pulmonary embolism due to tumor calcification and fragmentation. Retrospectively, therefore, the rationale for delaying surgical excision (low body weight, prematurity, expectation of spontaneous regression) appears unjustified. On the contrary, intracardiac masses with clinicopathologic features inconsistent with cardiac rhabdomyoma must be referred for early surgical excision to allow for diagnosis and prevention of life-threatening embolism. Furthermore, when fetal-type rhabdomyoma is identified in a child, genetic counseling must consider the possibility of occurrence of the tumor in future pregnancies and should advise in utero echocardiographic screening. In the case of diagnosis, however, any decision to interrupt pregnancy should take into consideration the fact that radical resection in infancy is feasible and no association with tuberous sclerosis has been reported.

\section{References}

1. Isaacs H. Fetal and neonatal cardiac tumors. Pediatr Cardiol. 2004;25:252-73.

2. Wu SS, Collins MH, de Chadarévian JP. Study of the regression process in cardiac rhabdomyomas. Pediatr Dev Pathol. 2002;5:29-36.

3. Schreiber C, Vogt M, Kostolny M, Gunther T, Lange R. Surgical removal of a rhabdomyoma in a neonate as rescue therapy. Pediatr Cardiol. 2006;27: $140-1$.

4. Reynolds C, Tazelaar HD, Edwards WD. Calcified amorphous tumor of the heart (cardiac CAT). Hum Pathol. 1997;28:601-6.

5. Hansen T, Katenkamp D. Rhabdomyoma of the head and neck: morphology and differential diagnosis. Virchows Arch. 2005;447:845-54.

\title{
Diagnosis and management of tritruncal heart in an infant
}

\author{
Glenn J. Pelletier, MD, ${ }^{\mathrm{a}, \mathrm{b}}$ Mary Sokoloski, MD, ${ }^{\mathrm{c}}$ and Richard Kardon, DO, ${ }^{\mathrm{c}}$ Philadelphia, Pa, and Miami, Fla
}

Ventriculoarterial connections are typically arranged with the aorta and main pulmonary artery (MPA) joined to the left ventricle (LV) and right ventricle (RV), respectively. Until 1982, when Díaz-Góngora and colleagues ${ }^{1}$ reported an autopsy case in which 3 separate arteries arose from the base of the ventricles, tritruncal heart was an unknown defect. The third artery was an intermediate trunk that arose from the LV and continued on as the right pulmonary artery (RPA). We report the diagnosis and management of the first known living child to undergo successful repair of a tritruncal heart.

\footnotetext{
From the Department of Surgery, Drexel University College of Medicine, ${ }^{\mathrm{a}}$ and the Section of Cardiothoracic Surgery, St Christopher's Hospital for Children, ${ }^{\mathrm{b}}$ Philadelphia, Pa, and the Department of Pediatrics, Division of Pediatric Cardiology,

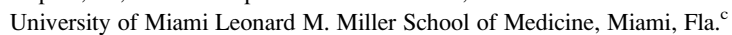
Received for publication Dec 4, 2007; accepted for publication March 13, 2008.

Address for reprints: Glenn J. Pelletier, MD, Section of Cardiothoracic Surgery, Saint Christopher's Hospital for Children, Erie Avenue at Front St, Philadelphia, PA 19134-1095 (E-mail: gpelleti@drexelmed.edu).

J Thorac Cardiovasc Surg 2009;137:753-5

0022-5223/\$36.00

Copyright (c) 2009 by The American Association for Thoracic Surgery

doi:10.1016/j.jtcvs.2008.03.057
}

\section{CLINICAL SUMMARY}

The patient was a male twin born at term. At 10 days after his birth, a heart murmur was detected. At presentation, the patient appeared well; however, the oxygen saturations recorded by noninvasive measurement varied from $83 \%$ to $97 \%$ when the patient was breathing ambient air. An echocardiogram demonstrated 3 arterial trunks originating from the base of the heart. The MPA $(11 \mathrm{~mm})$ arose from the $\mathrm{RV}$ and continued distally exclusively as the left pulmonary artery (LPA, $5 \mathrm{~mm})$. The aorta $(8 \mathrm{~mm})$ arose from the $\mathrm{LV}$ in the usual fashion. The third artery, the IT $(3 \mathrm{~mm})$, originated from the LV and continued as the RPA. At the base of the IT, a semilunar valve was seen through which antegrade flow into the RPA and regurgitant flow into the LV occurred (Figure 1,A). The RV pressure was estimated to be suprasystemic. There was predominantly right-to-left shunting through a patent foramen ovale. The LV was normal in size and function.

Cardiac catheterization confirmed the origin of the IT from the LV and its continuation as the RPA. Several aortopulmonary collaterals arose from the descending aorta to supply numerous segments of the right lung (Figure 1, $B$ ). Pressure measurements of the LV and RV were equivalent. 

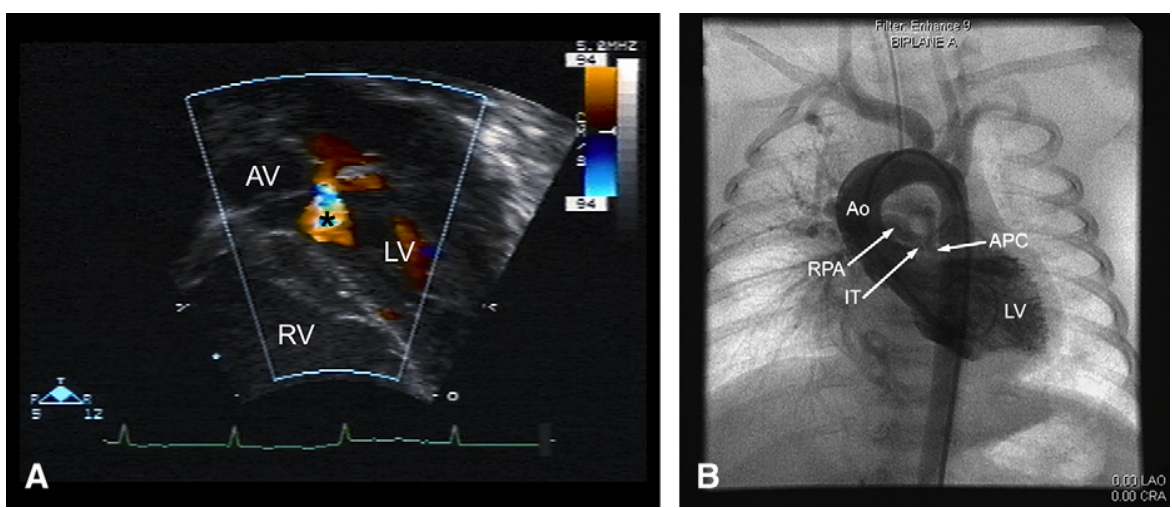

FIGURE 1. A, Transthoracic echocardiogram shows regurgitant flow (asterisk) through intermediate trunk into left ventricle $(L V)$. Aortic valve $(A V)$ is adjacent to but separate from this intermediate vessel. $R V$, Right ventricle. B, In left ventriculogram done through retrograde aortic catheter, left ventricle $(L V)$ ejects contrast-enhanced blood in usual fashion into aorta (Ao). Second vessel, intermediate trunk (IT), originates from base of left ventricle and gives rise to right pulmonary artery $(R P A)$, which supplies right lung. Aortopulmonary collateral $(A P C)$ vessel arises from proximal descending thoracic aorta and courses toward right lung. There is no pulmonary blood flow seen to left lung.

The pulmonary vascular resistance calculated for the left lung was 11 Wood units $/ \mathrm{m}^{2}$.

We obtained coronal views (Figure 2, A) and 3-dimensional reconstructions (Figure 2, B) of the heart and great vessels by computed tomographic scan. The spatial relationship of these 3 truncal arteries was IT-RPA anterior and leftward relative to the aorta but posterior and rightward relative to the MPA. The left coronary artery coursed immediately posterior to the IT. The RPA branching pattern was normal.

With the left lung receiving the full cardiac output and the right lung connected to the systemic circulation through the LV and several arterial collateral vessels, spontaneous abatement of the suprasystemic pulmonary vascular resistance and the cardiac volume overload was unlikely. We therefore chose to intervene. Our therapeutic strategy included dual surgical and interventional catheter approaches. On hospital day 8 , the patient underwent coil occlusion of the 3 principle aortopulmonary collaterals. During the next 4 days, the patient was supported with mechanical ventilation, precise fluid management, judicious inotrope use, and inhaled nitric oxide. Surgery was performed on hospital day 12 .

At the operation, a previously unrecognized patent ductus arteriosus was found between the LPA and the aorta and was ligated and divided. There was contiguity between the walls of the IT-RPA and the MPA but no luminal continuity. The base of the IT was divided above its attachment to the LV. The thin, rudimentary valve leaflet tissue within the root of the IT was oversewn. The shared wall between the ITRPA and the MPA was carefully divided. An end-to-side anastomosis of the native posterior walls of the RPA and the MPA was constructed, and the anterior wall was made with bovine pericardium.
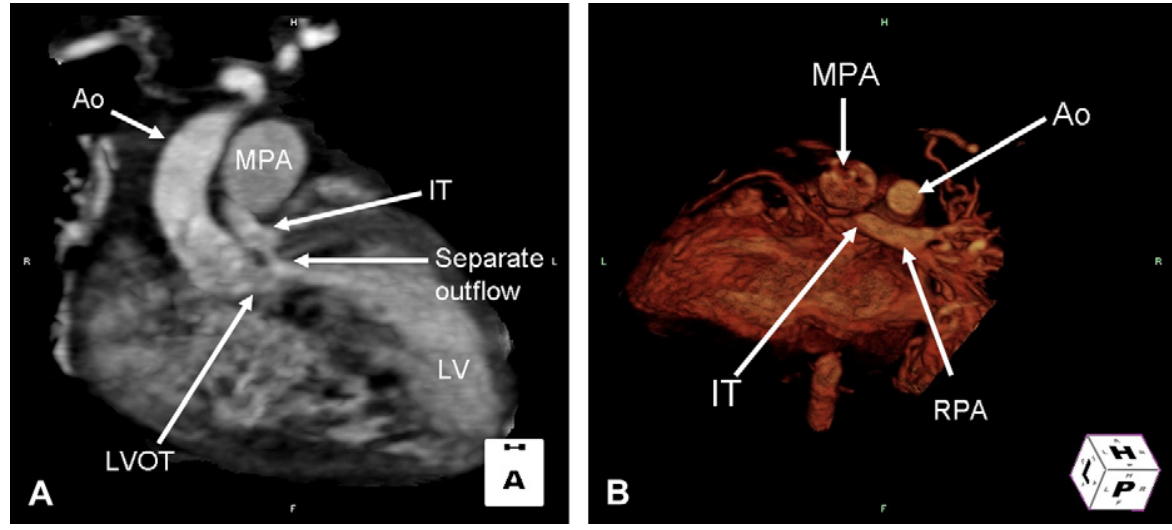

FIGURE 2. A, Contrast-enhanced, maximum-intensity projection computed tomogram of heart and great vessels. Image is viewed from anterior with $15^{\circ}$ degrees inferior angulation, as depicted by cube in lower right. Aorta $(A o)$ arises from left ventricular outflow tract $(L V O T)$. Through separate outflow from left ventricle $(L V)$, intermediate trunk $(I T)$ arises. Main pulmonary artery $(M P A)$ is anterior and leftward relative to intermediate trunk. B, Volume-rendered 3-dimensional computed tomographic image with cut plane to remove posterior structures. View is posterior with $36^{\circ}$ inferior and $24^{\circ}$ leftward angulation, as depicted by cube in lower right. Intermediate trunk arises from base of heart posterior and rightward in relation to main pulmonary artery and continues on as right pulmonary artery $(R P A)$, coursing posterior to aorta. 
The patient recovered fully and was discharged home with supplemental oxygen. An echocardiogram performed at discharge showed a hypertrophied RV with a flattened interventricular septum. RV pressure could not be estimated, with only trivial tricuspid regurgitation. Doppler flow pattern across both branch pulmonary arteries was mildly turbulent. In follow-up, the patient has undergone balloon angioplasty of moderate bilateral proximal branch pulmonary artery stenoses on two occasions. There are $15-\mathrm{mm} \mathrm{Hg}$ gradients across both branch pulmonary arteries. RV pressure is $50 \%$ systemic value at 13 months of follow-up. The patient currently continues to thrive without supplemental oxygen.

\section{DISCUSSION}

Tritruncal heart is a rare congenital heart malformation not previously described in a living individual. As such, there is no information available about the embryology, diagnosis, and treatment of this unusual anomaly. From our experience, however, we can offer insight into evaluating and managing such a case.

Although an embryologic explanation for tritruncal heart must remain speculative, we propose that the inciting event is failure of the proximal portions of the right and left VI arch vessels to fuse and form the pulmonary trunk, as has been proposed by Skidmore ${ }^{2}$ to occur normally. At surgery, finding the IT-RPA and MPA sharing a common vessel wall without luminal continuity is suggestive of this failure. Our finding of valve tissue, which we propose to represent a third semilunar valve, at the IT-LV junction is more difficult to explain. On the basis of theories of normal outflow tract and semilunar valve formation, ${ }^{3-5}$ we suspect that a complex interplay among the myocardial sleeve, the cardiac mesenchyme, and the endocardium of the developing outflow tract determines the genesis of semilunar valves from the conotruncal ridges during truncal septation. It is therefore possible that a right VI arch vessel that does not coalesce with its left counterpart to form a single pulmonary trunk could end up positioned over the LV, just millimeters away from its intended location. Here the RPA is still subject to the process of semilunar valve and outflow tract formation. The result could be an RPA originating as an IT from the LV with a semilunar valve intact.

We have learned key echocardiographic features that are discriminating for tritruncal heart. First, the presence of a diastolic regurgitant jet into the LV that does not originate from the aortic valve is suggestive. Second, this jet must be differentiated from one caused by an aorta-LV tunnel, in which the regurgitation occurs anterior and adjacent to the aortic valve but travels posterior to the infundibulum to enter the LV cavity. Third, demonstration of a valve distinct from the aortic valve that arises from the LV and connects to the RPA is a hallmark of the diagnosis. Supportive information includes an MPA that continues distally only as the LPA.

We developed a treatment strategy from the knowledge that this patient had suprasystemic pulmonary arterial pressure and volume loading of the heart produced by aortopulmonary collaterals. We first addressed the volume load by coil occluding the principle collaterals, with the intent of modifying the pathophysiologic condition to primarily pulmonary hypertension. Reducing collateral blood supply to the lungs also simplified intraoperative circulatory management and reduced the risk of pulmonary hemorrhage. Although the procedure was technically successful, the collateral occlusion and acute volume reduction of the LV produced a state of low cardiac output. Once the patient was in stable condition, the surgical repair to establish completely separate pulmonary and systemic circulations was straightforward. Although moderate bilateral branch pulmonary artery stenoses developed later, these narrowed vessels were satisfactorily treated with balloon dilations.

Tritruncal heart is a rare and fascinating heart malformation that occurs from a derangement in conotruncal development. Although this anatomy is unfamiliar, the diagnosis can be made by the distinguishing imaging features outlined here. The therapeutic approach should be guided by the pathophysiology, which is common to various other structural heart defects.

\section{References}

1. Díaz-Góngora G, Quero-Jiménez M, Espino-Velaj, Arteaga M, Bargeron L. A heart with three arterial trunks (tritruncal heart). Report of a case. Pediatr Cardiol. 1982;3:293-9.

2. Skidmore FD. Development of the right outflow tract and pulmonary arterial supply. Ann R Coll Surg Engl. 1975;57:186-97.

3. Timmerman LA, Grego-Bessa J, Raya A, Bertrán E, Pérez-Pomares JM, Díez J, et al. Notch promotes epithelial-mesenchymal transition during cardiac development and oncogenic transformation. Genes Dev. 2004;18:99-115.

4. Thompson RP, Fitzharris TP. Division of cardiac outflow. In: Ferrans VJ, Rosenquist G, Weinstein C, editors. Cardiac morphogenesis. New York: Elsevier; 1985. p. 169-80.

5. van Den Hoff MJ, Moorman AF, Ruijter JM, Lamers WH, Bennington RW, Markwald RR, et al. Myocardialization of the cardiac outflow tract. Dev Biol. 1999;212:477-90. 